\title{
Editorial
}

\section{Anesthesia human resources in Canada}

"It's hard to make predictions, particularly about the future"

Yogi Berra

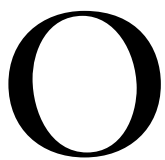

NTARIO has, and will continue to have, insufficient anesthesiologists to meet the demands of it's population. While this is probably a Canada-wide phenomenon, the article by Yang et al. ${ }^{1}$ provides a factual assessment of the magnitude of Ontario's problem. Action should be taken now to avert an anticipated crisis yet, to date, those making human resource decisions have largely ignored this specialty. Why?

Accurate physician resource planning depends upon the reliability of the data, and is easily challenged. Information about physician demographics and activities is usually generated from voluntary responses to specialty-initiated surveys, and is susceptible to criticism of bias. Beyond survey accuracy, it is difficult to decide what index drawn from the past will accurately describe the future needs of any specialty. ${ }^{2}$ Anesthesia, for example, is dependent upon the number of medical institutions, their medical as well as surgical programs, and the demographics of the patient population. ${ }^{3}$ Finally, there are alternative (non-specialist physicians) providers of anesthetic service in rural and smaller communities that influence the needed capacity of neighbouring larger centres served by specialists.

To define the needs of a certain specialty, several processes are available. ${ }^{4}$ In needs-based planning expert panels estimate the number of physicians of a given specialty required per capita; the predictions can be tainted by opinion as to who will provide service (for example: are alternative providers considered?), or how the process of care will occur in the future. In demand-based planning current utilisation patterns are used to indicate future workforce requirements; this assumes the current level of service is appropriate, a questionable position given the queues for elective operative services in Canada.

Unfortunately, the ability of the past to predict the future is always tenuous. The process of care may change: consider recent changes such as same-dayadmission surgery necessitating anesthetic clinics, or laparoscopic surgical advances made possible at considerable cost in anesthetic hours. Other factors may compound the calculations, such as anesthesiologists' life-style expectations and retirement plans. For example, the United Kingdom is also experiencing a shortage of anesthetists due to early retirements, attributed in part to changes in the registrar system (mandating closer on-call supervision by consultants), and a requirement for recredentialling. This follows upon an earlier UK study ${ }^{5}$ that identified anesthesia as a specialty with greater attrition from early retirement and deaths-in-post compared with other medical specialties. Australian anesthesiologists also retire earlier than other specialists. ${ }^{6}$ Accurate data on the expected career path of Canadian anesthesiologists will need to be gathered to project attrition from this specialty.

As an alternative to attempting to predict the future, bench-marking compares the current physician supply with that of another region, usually chosen because the determinants of health seem satisfactory in terms of quality and efficiency in that reference location. This method does not assume that it can predict the future, but only that the benchmarked area is currently well-positioned to deal with future needs. In primary care specialties the choice of the province against which to benchmark may change considerably the estimated physician resources, yet have no influence upon the population well-being. ${ }^{4}$ When the technique is applied to a secondary and facilitating specialty such as anesthesia, it is not known which population characteristics define equivalent specialist resource requirements. Community elements such as high birthing rates, trauma, or availability of alternative (non-surgical) therapy may be more important in

Address correspondence to: Peter G. Duncan MD FRCPC, Department of Anesthesia, Victoria General Hospital, 35 Helmcken Road, Victoria, B.C., V8Z 6R5 Phone: 250-727-4152; Fax: 250-727-4439; E-mail: pgd@home.com 
choosing an "anesthetic benchmark" than simple demographics of patient age and sex. Finally, whether a greater number of anesthesiologists can be supported in a given area is also speculative, for as soon as one factor ceases to limit operating room access another element (e.g.: nursing resources) takes its place. Bench-marking must, therefore, measure all components of a region's operating facilities.

But the issue remains unclear! Only a year before the current survey, the Chairman of the Ontario Hospital Restructuring Commission, Dr. Duncan Sinclair, ${ }^{7}$ suggested that "reducing the number of operating rooms would mean a decrease in demand for surgeons and anesthesiologists". Hospital closures would lead to the more rational provision of services, and eliminate procedures that provided no meaningful improvement in population health. About the same time, a prominent Canadian health policy advisor, Dr. R. G. Evans, pointed out ${ }^{8}$ that the projected requirement for physicians in Canada was flawed from the time of Medicare's introduction in the 1970s. The population boom of the post-war period has not been sustained, hence Canada now has too many physician mouths to feed because the population was overestimated by several million. Furthermore, he contends that all manpower planning is based upon "the key assumption, which is neither explicit nor justified, that the "need" for physicians cannot be less than the current supply, whatever that supply may be" (italics his). "Current service volumes or prevailing numbers of physicians are taken as minimum estimates of requirements, and various factors that might lead to an increase or decrease supply are identified and quantified, while potentially off-setting factors are left out of the account." He argues for a more directive physician management system that takes into account delivery system organisation and personnel substitution, elements that may (italics added) be part of a future medical system in Canada. As a final deflating comment to anesthesia's concern, he points out that medical payment policies in each province have been designed to limit the growth of service volumes; to react to threats of physician shortfalls would be, to put it mildly, inconsistent with public policy!

The control of financial costs of any health care system can be done in only two ways: through economic strategies that change the incentives within the system (market-place pressure, the USA model), or through regulation of resources. ${ }^{10}$ Canada has chosen to pursue the latter, but the politicalization of its medical services plans has made direct rationing of service to individual patients unacceptable. With no boundary definition of what constitutes "health services", and expectations of the consumer fed by the Americandominated media, Canadians have chosen secondary rationing wherein the use of services is limited by facility (including physician) availability.

Physicians are viewed as a national resource in Canada, and some feel they need to be "managed" to prevent overproduction and maldistribution. Through a series of deliberate steps, authorities began, in 1975, to limit physician immigration, and cut medical school enrolment by $17 \%$ in 1985 . They then recommended reducing graduate medical education training in primary care by 1991 , and in all specialties by $20 \%$ by 1994. ${ }^{9}$ More recently, the emphasis has shifted from national to provincial management of physician supply, with policies variably affecting internal migration in Canada, and limiting "re-entry" of graduated physicians from primary service to specialty training. The medical associations have been relatively complacent with these government policies, recognising that restricted entry at a time of capped budgets for medical services favours the incumbent members.

As we face a future shortfall of anesthesiologists in Canada, the deliberate consequence of public policy, should we simply accept it, and close operating rooms? Such a position may not be opportune for the specialty, for crisis management by administrators does not always lead to the desired result. In a political imperative to maintain surgical services, anesthetic initiatives outside the ORs (academic, research, and clinical) which have advanced the specialty's image, science, and value may have to be curtailed. Personal lifestyles important to the choice of this specialty may become jeopardized, leading to reduced recruitment and greater attrition. Finally, alternative models of service may be implemented in the absence of our ability to meet the service needs, devaluing the practice of this specialty.

We need to influence the opinion of those empow ered to make global decisions about the input to our specialty. The recent National Forum on Health ${ }^{10}$ concluded that the current level of combined public and private spending on health care in Canada is sufficient to support access to medically necessary services: we cannot expect more money to train a greater number of physicians. We must attract a greater percentage of the current medical school graduates. This is not easy because anesthesia residency positions are not always fully subscribed, ${ }^{1}$ and other specialties are also forecasting shortfalls. As has been done in Ontario, we must petition wherever and whenever we can, recognizing that evidence is not the sole factor that influences decision-making. The values and interests of the decision-makers as well as the context in 
which the decisions are made are also important. For example, when the National Forum on Health states as a priority ${ }^{10}$ "the health of Canadians will be best served by attending to the needs of children and families, by enhancing community action and by addressing the social and economic determinants of health", you know the road is uphill for anesthesia!

Within the specialty there is a need to show greater foresight in local planning of anesthetic practices. Only a few years ago there were no positions available in Canada for graduating residents: group anesthetic practices remained understaffed in the face of the uncertainty of "health care reform". A longer view would have seen flexible policies to retain Canadian graduates, and to keep anesthesiologists happy in their professional careers. There is a need to support lifestyle initiatives such as job sharing and sabbatical leaves as requested; in the long range it will keep a larger number of persons active within this profession. Finally, we should keep an open mind to concepts such as physician extenders that allow care for a greater number of patients with a constrained resource base.

In the end, the political decisions of recent years (limited immigration of qualified anesthesiologists, denial of re-entry from general practice, and reduction of the number of training positions in anesthesia) will either need to be changed, or we will have to change the manner in which medical services are provided. ${ }^{11}$ But, alas, policy makers do not read anesthesia journals! To persuade them requires objective information, gathered repeatedly over the years to show trends and directions, and which are substantiated by independent data banks. It may be their past decisions that got us into this position, but it is the specialty and the lifestyle of its members that will pay for inaction now!

\section{Ressources humaines en anesthésie au Canada}

«Il est difficile de faire des prédictions, en particulier à propos du futur»

Yogi Berra

L'Ontario a, et continuera d'avoir, une pénurie d'anesthésiologistes pour répondre aux demandes de sa pop ulation. Même si ce phénomène est probablement pancanadien, Yang et coll. ${ }^{1}$ ont choisi de nous présenter une évaluation factuelle de l'ampleur du problème ontarien. Il faut agir pour éviter une situation critique même si, jusqu'à maintenant, les décisions prises au sujet des ressources humaines ont le plus souvent oublié notre spécialité. Pourquoi?

La planification de ressources médicales suffisantes dépend de la fiabilité des données et peut être facilement contestée. Les données démographiques concernant les médecins, et leurs activités, proviennent habituellement des réponses volontaires à des enquêtes proposées par la spécialité et sont susceptibles de biais. Au delà de l'exactitude de l'enquête, il est difficile de décider quel sera l'indice, basé sur les années antérieures, pouvant décrire avec précision les besoins futurs de toute spécialité. ${ }^{2}$ L'anesthésie, par exemple, dépend du nombre d'institutions médicales, de leurs programmes médical et chirurgical et des caractéristiques démographiques de la population de patients. ${ }^{3}$ Enfin, certains médecins, non spécialisés, pratiquent l'anesthésie dans les régions rurales et les petites communautés, ce qui influence le nombre de médecins nécessaires dans de plus grands centres voisins desservis par des spécialistes.

Il existe différentes méthodes pour définir les besoins d'une spécialité. 4 Dans la planification basée sur les besoins, des groupes d'experts estiment le nombre de médecins d'une spécialité donnée nécessaires par habitant; les prédictions peuvent être modifiées par des questions, à savoir, qui va assurer les services? Peut-on compter, par exemple, sur des ressources alternatives? Comment vont se présenter les soins dans l'avenir? Dans la planification basée sur la demande, les modèles d'utilisation actuels sont utilisés pour indiquer les besoins futurs de main-d'oeuvre; cela suppose que les services sont présentement suffisants, ce qu'on peut mettre en doute étant donné les listes d'attente en chirurgie élective au Canada.

Malheureusement, il est toujours difficile de prédire l'avenir à partir du passé. La façon de fournir les soins peut changer : considérons les récents services de chirurgie d'un jour qui nécessitent des cliniques anesthésiques ou les progrès de la chirurgie laparoscopique rendus possibles au prix de nombreuses heures en services anesthésiques. D'autres facteurs peuvent se combiner aux calculs, comme les attentes des anesthésiologistes quant au style de vie et au régime de retraite. Par exemple, le Royaume-Uni est aussi confronté à une pénurie d'anesthésistes causée par les retraites anticipées, attribuables en partie à des modifications au système de résidence (exigeant une supervision sur demande, plus étroite par les spécialistes) et à une mise à jour obligatoire des connaissances. Cette situation confirme une étude ${ }^{5}$ antérieure du R-U qui identifiait l'anesthésie comme une spécial- 
ité où il y a un plus grand nombre de départs naturels, causés par les retraites anticipées et les décès en service, comparativement à d'autres spécialités médicales. Les anesthésiologistes australiens se retirent également plus tôt que les autres spécialistes. ${ }^{6}$ On doit rassembler des données exactes sur les projets de carrière des anesthésiologistes canadiens pour faire une projection des départs volontaires.

Pour suppléer à la tentative de prédire l'avenir, la mise en référence permet de comparer les effectifs médicaux actuels avec ceux d'une autre région choisie habituellement parce que la qualité et l'efficacité des soins de santé y semblent satisfaisantes. Cette méthode ne suppose pas qu'elle puisse prédire l'avenir, mais seulement que la région de référence soit actuellement bien préparée pour faire face aux besoins futurs. En ce qui concerne les spécialités de soins primaires, le choix de la province de référence peut changer considérablement l'estimation des ressources médicales, mais n'a pas d'influence sur le bien-être de la population. ${ }^{4}$ Par ailleurs, quand on ap plique la technique à une spécialité secondaire ou de soutien comme l'anesthésie, on ne sait quelles caractéristiques de la population servent à définir les demandes équivalentes de ressources médicales spécialisées. Des éléments relatifs à une collectivité comme le taux élevé des naissances, les traumatismes ou la possibilité de thérapie alternative à la chirurgie peuvent être plus importants au moment de choisir une référence anesthésique que les simples données démographiques comme l'âge et le sexe des patients. Enfin, le fait qu'un plus grand nombre d'anesthésiologistes puissent assurer des soins dans une région donnée relève aussi de la conjecture parce qu'aussitôt qu'un facteur cesse de limiter l'accès à la salle d'opération, un autre facteur entre en jeu, les ressources infirmières par exemple. Pour cette raison, la référence doit permettre de mesurer toutes les composantes des services chirurgicaux d'une région.

Le résultat demeure toutefois incertain! Un an seulement avant l'enquête actuelle, le président de la Com mission de restructuration hospitalière de l'Ontario, le docteur Duncan Sinclair, ${ }^{7}$ a émis l'hypothèse que «la réduction du nombre de salles d'opération pourrait signifier une baisse de la demande de chirurgiens et d'anesthésiologistes». La fermeture d'hôpitaux amènerait la distribution plus rationnelle des services et éliminerait les interventions qui n'améliorent pas la santé de la population de manière significative. À la même époque environ, un important conseiller canadien en matière de santé, le docteur R.G. Evans, a fait remarquer ${ }^{8}$ que l'estimation du nombre de médecins nécessaires au Canada n'était plus fiable comme au temps de l'introduction de Medicare dans les années 1970.
L'explosion démographique d'après-guerre ne s'est pas reproduite, si bien que le Canada a maintenant trop de médecins parce que la population a été surestimée de quelques millions. De plus, le docteur Evans prétend que toute planification de la main-d'oeuvre est basée sur «la prémisse-clé, qui n'est ni explicite ni justifiée, que les «besoins» de médecins ne peuvent être plus bas que les besoins actuels, quels que soient ces besoins» (l'italique est de lui). "Le volume actuel de services ou les effectifs médicaux en place présentement servent à l'estimation de besoins minimaux et différents facteurs qui pourraient faire augmenter ou chuter les effectifs sont identifiés et quantifiés, tandis que des facteurs potentiellement compensatoires ne sont pas pris en compte.» Il est en faveur d'une gestion plus directive des médecins qui tienne compte de l'organisation du système de répartition et de la substitution du personnel, éléments qui peuvent (italique ajouté) faire partie d'un futur système médical canadien. Comme dernier commentaire déflationniste concernant l'anesthésie, il fait remarquer que les modes de paiement médical de chaque province ont été conçus pour limiter l'augmentation de la quantité de services; réagir à la menace de pénurie de médecins serait, pour ne pas dire plus, en contradiction avec la politique nationale!

Le contrôle des dépenses de tout système de santé ne peut se faire que de deux façons : par des stratégies économiques qui modifient les mesures incitatives à l'intérieur du système (pression du marché, modèle américain) ou par la régulation des ressources. ${ }^{10} \mathrm{Le}$ Canada a choisi la deuxième voie, mais la politisation de ses services médicaux a provoqué un rationnement direct inacceptable des services aux patients en particulier. Sans une définition claire de ce qui constitue «des services de santé» et avec les attentes des citoyens entretenues par les médias dominés par les Américains, les Canadiens ont choisi la deuxième option où l'utilisation des services est limitée par la disponibilité des ressources, y compris les médecins.

Les médecins sont vus comme une ressource nationale au Canada, et certains pensent qu'ils doivent être «gérés» pour prévenir la surproduction et la mauvaise distribution. Les autorités ont commencé, en 1975, à limiter l'immigration de médecins et à réduire l'accès aux écoles de médecine pour en arriver, à la suite d'une série d'étapes calculées, à une baisse de 17 $\%$ en 1985. Puis, elles ont recommandé de réduire la formation spécialisée en soins primaires en 1991, et dans toutes les spécialités de $20 \%$ en $1994 .{ }^{9}$ Plus récemment, cette gestion nationale des ressources médicales est passée aux provinces et comporte des politiques qui touchent de façon variable la migration interne au Canada et limite le «retour» aux études, 
pour une spécialisation, des médecins diplômés en services primaires. Les associations médicales ont été relativement complaisantes envers ces politiques gouvernementales en reconnaissant que ces restrictions au moment où les budgets sont réduits pour les services médicaux favorisent les membres en place.

Comme nous faisons face à une pénurie prochaine d'anesthésiologistes au Canada, conséquence délibérée de la politique nationale, devons-nous l'accepter tout simplement et fermer les salles d'opération? Une telle décision peut être néfaste pour notre spécialité, la gestion de crise par des administrateurs ne produisant pas toujours les résultats escomptés. Devant l'urgence politique de maintenir les services chirurgicaux, il se pourrait qu'on doive réduire les initiatives anesthésiques en dehors des salles d'opération (université, recherches et cliniques), celles-là même qui ont fait progresser l'image, la science et la valeur de la spécialité. La qualité de vie personnelle, importante dans le choix de cette spécialité, pourrait être compromise, ce qui limiterait le recrutement et augmenterait les départs volontaires. Enfin, les modèles de remplacement des services pourraient être mis en application, puisque nous ne pouvons répondre aux besoins, dévaluant ainsi la pratique de l'anesthésiologie.

Nous devons influencer l'opinion de ceux qui ont le pouvoir de prendre des décisions globales concernant les besoins dans notre spécialité. Le récent Forum national sur la santé ${ }^{10}$ a conclu que le niveau actuel des dépenses combinées publiques et privées est suffisant pour conserver l'accès aux services médicalement nécessaires : nous ne pouvons espérer plus d'argent pour former un plus grand nombre de médecins. Nous devons attirer plus de diplômés des écoles de médecine. Ce n'est pas facile, car les postes de résident en anesthésie ne sont pas tous ouverts en permanence, ${ }^{l}$ et d'autres spécialités prévoient également des pénuries. Nous devons, comme en Ontario, faire des demandes partout et chaque fois que nous le pouvons, l'évidence n'étant pas le seul facteur qui influence la prise de décision. Les valeurs et les intérêts des décideurs, autant que le contexte dans lequel les décisions sont prises, sont aussi importants. Par exemple, quand on établit comme une priorité au Forum national ${ }^{10}$ "que la santé des Canadiens sera mieux assurée en répondant aux besoins des enfants et des familles, en encourageant l'action communautaire et en abordant les composantes sociales et économiques de la santé», on sait que ce sera une lutte permanente pour l'anesthésie!

Nous devons être plus prévoyants dans la planification locale des pratiques anesthésiques. Il y a quelques années seulement, aucun poste n'était ouvert au Canada pour les résidents diplômés : les groupes d'anesthésiologistes conservaient des postes non comblés à cause de l'incertitude de la «réforme des soins de santé». Une vision à plus long terme aurait permis des politiques flexibles pour retenir les diplômés canadiens et aurait gardé des anesthésiologistes heureux de leur carrière professionnelle. Il faut appuyer les initiatives en faveur de différents styles de vie comme le partage du temps de travail et les congés sabbatiques sur demande; à long terme, ces mesures retiendraient un plus grand nombre de personnes actives à l'intérieur de la profession. Finalement, nous devons faire attention à des concepts comme l'extension de la disponibilité médicale qui accorde des soins à un plus grand nombre de patients avec des ressources de base restreintes.

En fin de compte, les décisions politiques des dernières années (immigration limitée d'anesthésiologistes qualifiés, spécialisation refusée aux médecins généralistes et réduction du nombre de places de résidence en anesthésie) doivent changer ou nous devons changer la manière de fournir les services médicaux. ${ }^{11}$ Mais, hélas, les faiseurs de politiques ne lisent pas les journaux d'anesthésie! Il faut, pour les persuader, des informations objectives, rassemblées année après année pour indiquer les tendances et les orientations, et qui soient prouvées par des banques de données indépendantes. Ce sont peut-être leurs décisions passées qui nous ont placés dans cette position, mais c'est la spécialité et la qualité de vie de ses membres qui vont souffrir maintenant de l'inaction!

\section{References}

1 Yang H, Byrick R, Donen N. Analysis of anesthesia physician resources: projected Ontario deficit in 2005. Can J Anesth 2000; 47: 179-84.

2 Donen N, White IWC, Snidal L, Sammartin CA. Canadian anaesthesia physician resource planning - is it possible? Can J Anaesth 1995; 42: 348-57.

3 Roos NP, Bradley JE, Fransoo R, Shanahan M. How many physicians does Canada need to care for our ageing population? CMAJ 1998; 158: 1275-84.

4 Roos NP. Physician resource planning: ways and means. Can Med Assoc J 1997; 157: 1229-30.

5 McNamee R, Keen RI, Corkill CM. Morbidity and early retirement among anaesthetists and other specialists. Anaesthesia 1987; 42: 133-40.

6 Theile DE, Brennan P, Gavel P, Harding J, Horvath J. Methodology for the study and projection of surgical and anaesthetic workforce. Aust N Z J Surg 1998; 68: 481-92.

7 Gray C Will hospital closures mean physician unemployment in Ontario? Can Med Assoc J 1997; 156: 1614-6. 
8 Evans RG. New bottles, same old wine: right and wrong on physician supply. Can Med Assoc J 1998; 158: 757-9.

9 Sullivan RB, Watanabe M, Whitcomb ME, Kindig DA. The evolution of divergences in physician supply policy in Canada and the United States. JAMA 1996; 276 : 704-9.

10 Watanabe M. A call for action from the National Forum on Health. Can Med Assoc J 1997; 156: 999-1000.

11 Dauphine WD. Medical workplace policy making in Canada: are we creating more problems for the future? Clin Invest Med 1996; 19: 286-91. 